\title{
Nuevo logro para Analecta Veterinaria
}

Con grato placer deseamos comunicar a todos los lectores que nuestra revista, ANALECTA VETERINARIA, ha sido seleccionada para integrar el Núcleo Básico de Revistas Científicas Argentinas (NBR), un proyecto de CONICET (Consejo Nacional de Investigaciones Científicas y Técnicas) con sede en el CAICYT (Centro Argentino de Información Científica Tecnológica).

El NBR nuclea a un conjunto de publicaciones científicas y tecnológicas argentinas de excelencia, en los distintos campos del conocimiento. Dichas publicaciones científicas son sometidas a una evaluación exhaustiva con criterios únicos definidos de calidad y trascendencia, acorde con criterios internacionales.

La inclusión de ANALECTA VETERINARIA en el NBR permitirá una mayor visualización de nuestra revista tanto en la esfera nacional como internacional. Asimismo, permitirá la posibilidad de aplicar para su inclusión en otros portales, como SCIELO (Scientific Electronic Library Online), un modelo para la publicación electrónica cooperativa de revistas científicas en Internet, especialmente desarrollado para responder a las necesidades de la comunicación científica en los países en desarrollo y particularmente de América Latina y el Caribe.

Queremos agradecer a todos nuestros autores del pasado y presente por haber confiado en la calidad científica de ANALEcta Veterinaria. Esperamos que esta reciente inclusión nos permita incrementar la cantidad de trabajos provenientes de distintas partes del mundo, para poder llevar el conocimiento científico hacia todos los rincones del planeta.

Consejo Editorial

ANALECTA VETERINARIA

Analecta Vet 2019; Enero-Junio; 39(1):1

Impresa ISSN 03655 14-8 Electrónica ISSN 1514-2590

doi.org/10.24215/15142590eo36 